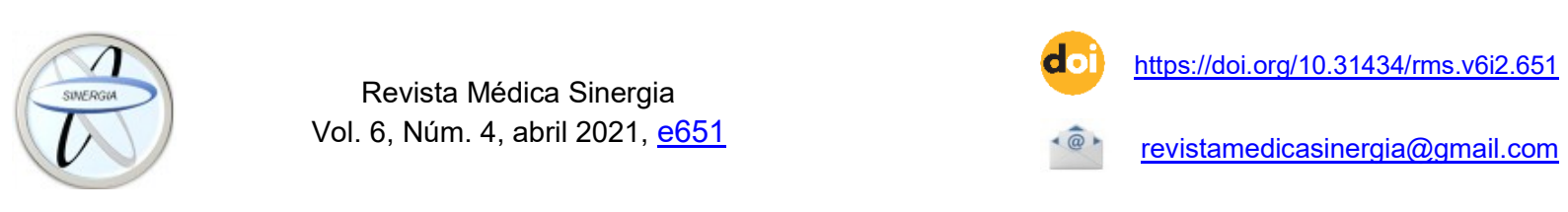

\title{
Miastenia gravis: fisiopatología y manejo perioperatorio
}

Myasthenia gravis: pathophysiology and perioperative management

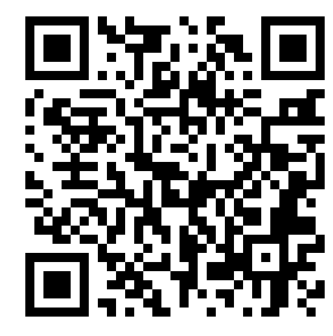

Recibido 03/02/2021

\author{
${ }^{1}$ Dr. Kevin Daniel Hines Chaves \\ Investigador independiente, San José, Costa Rica \\ (iD) https://orcid.org/0000-0002-6949-3179
}

${ }^{2}$ Dra. Mariela Gutiérrez Núñez Investigadora independiente, San José, Costa Rica

(iD) https://orcid.org/0000-0002-0663-0404

${ }^{3}$ Dra. Valeria Tinoco Chavarría Hospital Calderón Guardia, San José, Costa Rica (D) https://orcid.org/0000-0002-5759-9881

Aceptado

23/02/2021

\section{RESUMEN}

La miastenia gravis es una enfermedad autoinmue que afecta la únion neuromuscular, mediante el bloqueo funcional de la misma, al producirse anticuerpos contra receptores nicotínicos. Se presenta con síntomas de fatiga de diferentes grupos musculares, que puede llegar a afectar el habla, la deglución y la respiración. Esta patología se trata con medicamentos anticolinesterásicos, coticosteroides y/o timectomía. Los pacientes con miastenia gravis presentan mayor riesgo de complicaciones perioperatorias que la población general. Debido a esto se deben tener especiales cuidados en el perioperatorio. El fallo respiratorio es una complicación potencialmente mortal, por lo que se deben minimizar los riesgos de debilidad de los múaculos respiratorios.

PALABRAS CLAVE: miastenia gravis; enfermedades musculares; atención perioperativa; inhibidores de la colinesterasa; timectomía.

\footnotetext{
ABSTRACT

Myasthenia Gravis is an autoimmune disease that affects the neuromuscular junction, by blocking it functionally, by producing antibodies against nicotinic receptors. It presents with symptoms of fatigue in different muscle groups, which can affect speech, swallowing and breathing. This pathology is treated with anticholinesterase drugs, corticosteroids and/or thymectomy. Patients with myasthenia gravis have a higher risk of perioperative complications than the general population. Due to this, special care must be taken in the perioperative period. Respiratory failure is a life-threatening complication, so the risks of respiratory muscle weakness should be minimized. the patient is aware of possible complications
} 
KEYWORDS: myasthenia gravis; muscular diseases; perioperative care; cholinesterase inhibitors; thymectomy.

${ }^{1}$ Médico general, graduado de la Universidad de Costa Rica (UCR), cód. MED15872. Correo: kevin.hines19@gmail.com

${ }^{2}$ Médica general, graduada de la Universidad de Costa Rica (UCR). cód. MED15869. Correo: marigutierrez95@gmail.com

${ }^{3}$ Médico residente de Cirugía General, graduada de la Universidad de Costa Rica (UCR). Correo: valetinoco23@gmail.com

\section{INTRODUCCIÓN}

La miastenia gravis (MG) es un trastorno autoinmunitario de la unión neuromuscular mediado por autoanticuerpos contra el receptor nicotínico de acetilcolina y la tirosina quinasa específica del músculo (1). Esto resulta en una disminución del número de unidades activas, y provoca bloqueo funcional de la unión neuromuscular que se manifiesta por debilidad y fatiga de los músculos voluntarios con mejoría después del reposo (2-4).

Se revisa este tema debido a que, por la naturaleza de esta patología, se tienen que tener especiales cuidados en el manejo perioperatorio. Se ha reportado que, en comparación con los pacientes quirúrgicos sin MG, los pacientes quirúrgicos con MG presentan mayores riesgos de neumonía posoperatoria, septicemia, sangrado posoperatorio y complicaciones en general (5).

Es importante destacar que la revisión trata el manejo perioperatorio de pacientes que padecen MG independientemente de cual sea la indicación quirúrgica, no está enfocada en la timectomía. Existe abundante bibliografía sobre las implicaciones de la $M G$, en la timectomía, no así en las generalidades durante cualquier otra indicación quirúrgica que tenga un paciente con MG. Además, cuando se trata de información disponible en castellano, esta es aún más limitada.

El objetivo de esta revisión bibliográfica es detallar la patogenia, y con mayor énfasis, el manejo perioperatorio en pacientes con MG. Asimismo, se decide incluir parte de la sintomatología, diagnóstico, clasificación y tratamiento de la MG, para así contextualizar de mejor manera el tema tratado.

\section{MÉTODO}

Para la elaboración de esta revisión bibliográfica se realizó una búsqueda de referencias en las bases de datos PUBMED y EBSCOhost Academic Search Ultimate, mediante el Sistema de Bibliotecas, Documentación e Información de la Universidad de Costa Rica, utilizando los términos de búsqueda "perioperative", "myasthenia gravis" AND "surgery" y "pathophysiology" AND "myasthenia gravis"; con el objetivo de encontrar artículos que se enfocaran en el manejo que se le debe dar a pacientes que padecen MG en el perioperatorio. Se incluyó bibliografía en idioma inglés y español, publicada entre los años 2015 a 2021 (enero). Posteriormente se evaluó la información recopilada y se procedió a redactar esta revisión.

\section{EPIDEMIOLOGÍA}

La MG es un trastorno autoinmune que afecta aproximadamente a 15 personas por cada 100.000 (1). Puede presentarse a cualquier edad, aunque es más común en adultos jóvenes y de mediana edad (6). Aproximadamente el $10 \%$ de los pacientes son niños y adolescentes. Existe preponderancia femenina en la MG de inicio temprano (EOMG, por sus siglas en inglés) y masculina en la MG de inicio tardío (LOMG, por sus siglas en inglés) $(1,6)$. Además, se 
ha visto un aumento en la prevalencia e incidencia de LOMG en todo el mundo (6).

\section{FISIOPATOLOGÍA}

Como ya se mencionó la MG es un trastorno autoinmunitario de la unión neuromuscular mediada por autoanticuerpos, específicamente contra el receptor nicotínico de acetilcolina o contra proteínas vecinas implicadas en la agrupación de acetilcolina $(1,7)$. Debido a que estos anticuerpos son específicos para los receptores de acetilcolina (AchR) nicotínicos, los AChR en el corazón, la vejiga y el intestino no se ven afectados (3).

La unión neuromuscular no tiene una barrera hematoencefálica, por lo que los anticuerpos atacan fácilmente los componentes. El anticuerpo más común que se encuentra en pacientes con $M G$ se dirige contra el receptor de acetilcolina (AChR) y se encuentra en $85 \%$ de los pacientes con MG generalizada y $50 \%$ de los pacientes con MG ocular.

Existen diferentes tipos de anticuerpos $(1,8)$. Los anticuerpos AChR pertenecen mayoritariamente a las subclases lgG1 e IgG3, que activan la cascada del complemento, provocando la pérdida de AChR de la unión neuromuscular por 3 mecanismos: daño activado por complemento, modulación antigénica y bloqueo directo de la función. Para compensar la disminución de la transmisión neuromuscular causada por la pérdida de $A C h R$, hay un aumento en el contenido cuántico de $\mathrm{ACh}$ en $\mathrm{MG}$ con anticuerpos positivos. Por el contrario, los anticuerpos contra la quinasa específica de músculo (MuSK) pertenecen principalmente a la subclase IgG4, que no activan el complemento: por lo tanto, parece improbable que el daño activado por el complemento juegue un papel en MG asociada a MuSK (MuSK-MG). Los anticuerpos MuSK disminuyen la densidad postsináptica de los AChR y alteran su alineación entre la terminal nerviosa y la membrana postsináptica (8). MuSK-MG tiene características clínicas únicas, como debilidad bulbar prominente, atrofia muscular y preservación relativa de los músculos oculares (1).

Hay un creciente número de informes que describen anticuerpos circulantes contra otras proteínas de la placa terminal en MG. Estos incluyen la proteína 4 relacionada con el receptor de lipoproteínas de baja densidad; los anticuerpos se unen a esta proteína de membrana in vivo, bloqueando la interacción de la proteína 4 relacionada con el receptor de lipoproteínas de baja densidad y la agrina y, por lo tanto, también inhibiendo el agrupamiento de AChR en la membrana. Otras proteínas dirigidas por los anticuerpos asociados a MG son agrina (esencial para la función de AChR), rapsina y, más recientemente, cortactina (1).

Las células epiteliales neoplásicas expresan de forma variable epítopos de antígenos estriacionales, que incluyen titina (que mantiene la flexibilidad de la estructura de la célula muscular) y rianodina (un canal de calcio del retículo sarcoplásmico que media la contracción de la célula muscular). Los anticuerpos contra estas dos proteínas se presentan en algunos pacientes con MG asociada a AChR con timoma, y esto se considera un marcador de MG paraneoplásica. Se ha encontrado que los subgrupos de MG con diferentes autoanticuerpos, con o sin timoma, tienen características clínicas relativamente distintas y responden de manera diferente a la inmunoterapia estándar (1).

\section{SÍNTOMAS}

Los síntomas de MG generalmente se caracterizan por síntomas oculares, bulbares y sistémicos (3). Las características de presentación de los pacientes con MG pueden incluir las 
siguientes: músculos voluntarios fatigados que afectan los músculos extraoculares del ojo (diplopía) y los párpados (ptosis), síntomas bulbares que incluyen dificultad para masticar y tragar (disfagia), debilidad generalizada leve a grave, dificultad para hablar y falla respiratoria (4).

En el $15 \%$ de todos los pacientes con MG, los síntomas y signos se limitan a los músculos oculares. Solo la mitad de los pacientes con MG ocular tienen anticuerpos musculares detectables. La ptosis y la diplopía son síntomas iniciales comunes, pero la enfermedad permanece restringida a los músculos oculares solo en una minoría de pacientes. En el $90 \%$ de los pacientes que continúan teniendo $M G$ puramente ocular 2 años después del inicio de los síntomas, la enfermedad persistirá como una debilidad focal del músculo ocular y nunca se generalizará (9).

\section{DIAGNÓSTICO}

El diagnóstico de MG se confirma por la presencia de anticuerpos contra AChR, MuSK $u$ otros autoanticuerpos antes mencionados en individuos con síntomas compatibles con la enfermedad (1).

Se sospecha MG en pacientes con antecedentes y signos de debilidad fluctuante de los músculos voluntarios, que empeora con el esfuerzo y mejora en reposo. La confirmación del diagnóstico se logra luego mediante: a) detección de anticuerpos séricos; b) estudios de electromiografía (EMG) que muestran una disminución del potencial de acción del músculo compuesto en la estimulación nerviosa repetitiva de baja frecuencia o un aumento de la fluctuación en la EMG de fibra única; c) respuesta clínica a los inhibidores de la acetilcolinesterasa. Los resultados positivos en b) y c) confirman un defecto postsináptico de transmisión neuromuscular, mientras que la detección de anticuerpos específicos establece el diagnóstico de MG (10).
Los anticuerpos anti-AChR, la causa directa de los síntomas, son específicos de MG y rara vez se detectan en personas por lo demás sanas. Sin embargo, los títulos absolutos de estos anticuerpos no se correlacionan bien con la gravedad de la MG. Además, entre el 10 y el $15 \%$ de los pacientes con MG son negativos para los anticuerpos $A C h R$, que se denomina $M G$ seronegativa (SNMG) (3).

Además de utilizar el perfil de autoanticuerpos, la MG se puede clasificar según la ubicación de los músculos afectados (oculares vs generalizados), la edad de inicio de los síntomas y la naturaleza de la patología tímica (1).

\section{CLASIFICACIÓN}

Los síntomas clínicos se pueden evaluar con base en la clasificación de Osserman (Tabla 1), así como por la clasificación de la Myasthenia Gravis Foundation of America (MGFA). La gravedad se evalúa utilizando sistemas de puntuación como la puntuación de MG cuantitativa para la gravedad de la enfermedad (puntuación de QMG) y el perfil de actividades de la vida diaria de MG (MGADL) (3).

\section{TRATAMIENTO}

Las modalidades de tratamiento para la MG incluyen, tratamiento medicamentoso (que engloba el empleo de fármacos que inhiben la acetilcolinesterasa, así como el uso de corticosteroides, como terapia inmunosupresora), el tratamiento quirúrgico (teniendo como representante a la timectomía), por último, la plasmaféresis como tratamiento para crisis en casos de MG grave.

Todos los subgrupos de MG responden a la inhibición de la acetilcolinesterasa. Las anticolinesterasas inhiben la enzima acetilcolinesterasa que aumenta la porción de ACh disponible para la contracción 
muscular en la unión neuromuscular. La piridostigmina es el fármaco preferido para el tratamiento de los síntomas en todos los subgrupos de MG. La neostigmina y el cloruro de ambenonio también son inhibidores de la acetilcolinesterasa, pero son menos eficaces que la piridostigmina en la mayoría de los pacientes. La anticolinesterasa fisostigmina no se usa ya que esta droga atraviesa la barrera hematoencefálica y produce efectos no deseados en el sistema nervioso central (4, 9).

En el caso de que la terapia con medicamentos anticolinesterasa resulte ineficaz, se considera la terapia inmunosupresora con corticosteroides (4). El consenso de expertos y los datos de ensayos controlados limitados apoyan el uso de prednisona o prednisolona en combinación con azatioprina como tratamiento de primera línea. La prednisona y la prednisolona se consideran igualmente eficaces (9). En la mayoría de los pacientes, la azatioprina se agrega a la prednisolona porque esta combinación proporciona un mejor resultado funcional con menos efectos secundarios que la monoterapia con prednisolona. Si los glucocorticoides están contraindicados o si el paciente los rechaza, la azatioprina puede administrarse sola (9). Estudios sobre corticosteroides han revelado que en la $M G$ se produjo una remisión, una mejoría notable o un riesgo reducido de conversión de la forma ocular a la generalizada, lo que aumentó la inclinación a usar estos fármacos a pesar de la ausencia de ensayos controlados aleatorizados (11).

La terapia debe diseñarse para mejorar las características clínicas rápidamente y mantener los síntomas controlados a largo plazo. Se puede lograr una mejora rápida cuando sea necesario, mediante la administración de inmunoglobulina intravenosa o plasmaféresis. La plasmaféresis se reserva para el tratamiento de la MG grave, por lo general en el contexto de una crisis. Es útil como recurso temporal en pacientes gravemente afectados o para mejorar la condición del paciente antes de la cirugía (7).

\section{TIMECTOMÍA}

Para decidir sobre las indicaciones quirúrgicas y evaluar el estado de MG perioperatoria, son importantes las entrevistas con el médico sobre los hallazgos físicos (3).

La glándula del timo está involucrada en la función inmunológica y se cree que finalmente produce anticuerpos AChR responsables de MG (4). El $10 \%$ de los pacientes con MG tienen un timoma y la prevalencia aumenta con la edad (9). En el sentido contrario, la MG afecta aproximadamente entre 30 y $40 \%$ de los pacientes con timoma y representa el síndrome paraneoplásico más común (12). Otros estudios previos indican como estadística que entre 24,5 y el $40 \%$ de los pacientes con timoma desarrollan MG y entre 15 y el $20 \%$ de los pacientes con MG se diagnostican con timoma. Los timomas ocurren con frecuencia en pacientes masculinos mayores de 40 años al inicio de la MG (13)

La timectomía, la extirpación quirúrgica de la glándula del timo, se realiza con el objetivo de inducir la remisión del proceso de la enfermedad o al menos permitir la reducción de las dosis de inmunosupresores requeridas por los pacientes con MG (4). Un ensayo aleatorizado mostró que la timectomía transesternal prolongada podría mejorar los resultados clínicos durante un período de 3 años en pacientes con MG no timomatosa. La escisión radical de la neoplasia con todo el tejido del timo también está indicada en pacientes con timoma con MG. El pronóstico neurológico a largo plazo de la timectomía extendida en estos 
pacientes, y en los que no presentan timoma, es similar (13).

Los pacientes con MG seronegativa (SNMG) no suelen estar asociados con timoma, y alrededor del $50 \%$ de ellos son positivos para anticuerpos anti-MuSK. La mayoría de los pacientes con SNMG con anticuerpos anti-MuSK positivos no tienen hiperplasia tímica, por lo que no hay una indicación agresiva para timectomía. Asimismo, debido a que los pacientes con MG de tipo ocular a veces se resuelven espontáneamente, no existe una indicación agresiva para la timectomía (3).

\section{MANEJO PERIOPERATORIO}

Como ya se mencionó, los pacientes con MG suelen tratarse con agentes anticolinesterásicos, prednisona e inmunosupresores. Algunos pacientes pueden requerir terapia de intercambio de plasma o inmunoglobulina intravenosa en crisis aguda y rápido deterioro de los síntomas (6).

Se sabe que varios fármacos exacerban la MG (tabla 2) y algunos de estos fármacos pueden tener un uso perioperatorio potencial.

La preparación preoperatoria es fundamental en pacientes con MG. Se deben realizar estudios de función pulmonar porque la enfermedad respiratoria crónica y una capacidad vital preoperatoria $<2,9 \mathrm{~L}$ son 2 criterios predictivos de soporte respiratorio postoperatorio (6).

Los pacientes con MG pueden tener poca reserva respiratoria y los fármacos que pueden deprimir las funciones respiratorias deben usarse con precaución y evitarse en pacientes con síntomas bulbares. Dichos pacientes pueden premedicarse con atropina y se puede usar diazepam para sedación. Es una práctica usual retener la piridostigmina en la mañana de la cirugía para evitar los efectos secundarios muscarínicos y la necesidad de un relajante muscular, pero el valor de retener piridostigmina es cuestionable debido a las posibles complicaciones respiratorias que plantea. Los pacientes que toman preparaciones de piridostigmina de acción prolongada pueden recibir una formulación de acción corta la noche anterior a la cirugía (6).

\begin{tabular}{|l|l|}
\hline \multicolumn{2}{|l|}{ Tabla 1. Clasificación de Osserman } \\
\hline I & Miastenia ocular \\
\hline IIA & $\begin{array}{l}\text { Miastenia generalizada leve. Progresión } \\
\text { lenta: no crisis, respuesta a tratamiento. }\end{array}$ \\
\hline IIB & $\begin{array}{l}\text { Miastenia generalizada moderadamente } \\
\text { severa: afectación esquelética y bulbar } \\
\text { severa, pero sin crisis. Respuesta menos } \\
\text { satisfactoria a tratamiento }\end{array}$ \\
\hline III & $\begin{array}{l}\text { Miastenia aguda fulminante. Progresión } \\
\text { rápida de síntomas severos y pobre } \\
\text { respuesta a tratamiento. }\end{array}$ \\
\hline IV & $\begin{array}{l}\text { Miastenia grave tardía. Igual a III pero } \\
\text { con progresión al menos 2 años después } \\
\text { de las clases I y II }\end{array}$ \\
\hline $\begin{array}{l}\text { Fuente. Adaptado de Kadota Y, Horio H, Mori T, } \\
\text { Sawabata N, Goto T, Yamashita S-I, et al. } \\
\text { Perioperative management in myasthenia gravis: } \\
\text { republication of a systematic review and a proposal } \\
\text { by the guideline committee of the Japanese } \\
\text { Association for Chest Surgery 2014. Gen Thorac } \\
\text { Cardiovasc Surg. 2015;63(4):201-15. } \\
\text { https://doi.org/10.1007/s11748-015-0518-y }\end{array}$ \\
\hline
\end{tabular}

Es importante que a los pacientes no les falten sus medicamentos para la MG en el perioperatorio (14). Después de la operación, si no se extuban o si la administración oral no es posible, se debe considerar la sustitución parenteral o con sonda nasogástrica; puede ser necesario ajustar la dosis $(6,14)$. Cuando se administra por vía intramuscular, se administra $1 / 10$ de la dosis habitual, y cuando se administra por vía intravenosa, se administra $1 / 30$ de la dosis habitual (6).

La monitorización intraoperatoria con electrocardiograma, monitorización de la presión arterial, oximetría de pulso, CO2 espiratorio final $y$ análisis de gases espiratorios están justificados en pacientes con MG. La transmisión neuromuscular se 
puede controlar durante la cirugía mediante la estimulación de nervios periféricos para ajustar la dosis necesaria de relajantes musculares y asegurar la reversión adecuada del bloqueo neuromuscular al final de la cirugía (6).

El uso de anestesia regional o local es justificado siempre que sea posible. La anestesia general se puede realizar de forma segura en pacientes que están óptimamente preparados y la transmisión neuromuscular se controla adecuadamente durante y después de la cirugía (6). Se han realizado estudios comparando la anestesia generalepidural (AGE) y la anestesia general sola (AG), los mismos muestran como el empleo de AGE requirie menos tiempo de extubación y recuperación postoperatoria, a la vez que la estabilización hemodinámica perioperatoria por esta vía, reduce el consumo de anestésicos generales, y acorta el tiempo de extubación (15).

Todos los pacientes con MG deben ser monitoreados de cerca en el posoperatorio en la unidad de cuidados postanestésicos o en la unidad de cuidados intensivos quirúrgicos, donde se puede restablecer inmediatamente la asistencia respiratoria. El control adecuado del dolor posoperatorio, el baño pulmonar y la evitación de fármacos que interfieren con la transmisión neuromuscular facilitarán la extubación traqueal temprana (6).

Junto con los medicamentos anticolinesterásicos, los esteroides a menudo se administran antes de la cirugía (3). El paciente que recibe dosis más altas de esteroides puede necesitar dosis extra de esteroides perioperatorios (14). Las graves preocupaciones sobre la administración preoperatoria masiva de esteroides son un retraso en el momento de la operación y el desarrollo de efectos adversos del esteroide en el período perioperatorio, como infección y cicatrización deficiente de la herida. Se recomienda iniciar esteroides en dosis bajas, con un aumento gradual o dosis en días alternos. Se ha informado que el empeoramiento inicial de la MG por los esteroides ocurre entre 25 y $75 \%$ de los pacientes. Si bien los efectos adversos varían con las dosis de esteroides y la duración de la administración, ocurren a una tasa entre 38 y $67 \%$ (3).

En cuanto al periodo postoperatorio, la complicación más común entre los pacientes con MG es la insuficiencia respiratoria. La monitorización neuromuscular continua detectaría cualquier parálisis muscular residual por rocuronio que podría revertirse rápida y completamente con sugammadex (14).

Sugammadex es una c-ciclodextrina modificada que revierte el efecto de los agentes bloqueadores neuromusculares esteroides no despolarizantes rocuronio y vecuronio. El uso de agentes bloqueadores neuromusculares es controvertido en pacientes con MG. En general, las técnicas anestésicas no relajantes musculares en pacientes con AChR-MG parecieron ser seguras. Por otro lado, el rocuronio podría resultar útil tras la introducción de sugammadex como antagonista selectivo. Se ha observado una rápida recuperación de la función neuromuscular en pacientes con AChR-MG que reciben rocuronio cuando se utiliza sugammadex (2 $\mathrm{mg} / \mathrm{kg})$ para la reversión (8). El sugammadex intravenoso resulta en una recuperación rápida y predecible del bloqueo neuromuscular moderado y profundo en pacientes sometidos a cirugía que reciben rocuronio o vecuronio (16). La combinación de rocuronio y sugammadex podría ser una opción alternativa para que los pacientes con MG eviten la acción prolongada de los agentes bloqueadores neuromusculares, especialmente cuando el bloqueo neuromuscular es obligatorio durante la cirugía (8).

\section{CRISIS MIASTÉNICA}


La crisis miasténica posoperatoria es una afección potencialmente mortal, caracterizada por una exacerbación aguda de los síntomas de MG condebilidad muscular que requiere intubación o extubación retrasada después de la cirugía. La identificación de los pacientes de alto riesgo es entonces útil para que el anestesiólogo planifique el curso postoperatorio, particularmente en lo que respecta a la duración de la intubación y el ingreso en la unidad de cuidados intensivos. Se han detectado diferentes factores como predictores de crisis miasténica posoperatoria. Comacchio et al hacen alusión a que Leuzzi y sus colegas desarrollaron una puntuación predictiva para la crisis miasténica posoperatoria basada en el estadio de Osserman, la duración de los síntomas miasténicos, el índice de masa corporal y la asociación con las resecciones pulmonares (12).

Las crisis incluyen crisis miasténica debido al empeoramiento de la propia MG y crisis colinérgica debido a una sobredosis de fármacos anticolinesterásicos. diagnóstico diferencial puede requerir una prueba de edrofonio. Los pacientes a los que no se les puede retirar el soporte ventilatorio incluso unos días después de la cirugía suelen estar en crisis miasténica (3).

Un criterio para uso de ventilación mecánica (VM) en la exacerbación de MG es una capacidad vital inferior a $15-20 \mathrm{ml} / \mathrm{kg}$ de peso corporal. Sin embargo, los cambios de la condición respiratoria o de sintomatología bulbar deben evaluarse para determinar la indicación de VM. Para la VM, se recomienda la ventilación con presión positiva para prevenir el colapso de los alvéolos pulmonares y el desarrollo de atelectasias. Los fármacos anticolinesterasa no son necesarios durante la ventilación mecánica y, por lo general, se suspenden. Con esta pausa del fármaco, se puede esperar que aumente la sensibilidad a los fármacos anticolinesterasa. La recuperación de los músculos respiratorios es necesaria para el destete de los soportes ventilatorios (3).

\section{CONCLUSIONES}

Como se documentó en esta revisión, la MG es una patología autoinmune con especiales implicaciones en el manejo perioperatorio para los pacientes que la padecen.

En la revisión realizada se detalla la patogenia de la enfermedad y el manejo perioperatorio de los pacientes con MG con cualquier indicación quirúrgica en general, con lo cual se espera haber logrado el objetivo propuesto, al ampliar y actualizar el conocimiento sobre la MG y sus implicaciones en cuanto al manejo en el periodo perioperatorio.

En conclusión, se necesita una planificación cuidadosa para los pacientes con MG sometidos a cirugía. Ciertos medicamentos que se usan antes, durante o después de la operación pueden exagerar y empeorar los síntomas de la MG. Se debe tratar de que los pacientes interrumpan en la menor medida posible su medicación contra la MG, para lo que se cuenta con diferentes medidas. Los pacientes con MG presentan un mayor riesgo anestésico y complicaciones relacionadas con el perioperatorio. Los proveedores de atención perioperatoria deben intentar minimizar el riesgo de debilidad de los músculos respiratorios y la consiguiente falla respiratoria, la cual es una complicación potencialmente mortal. Por último, es importante que tanto el personal que atiende al paciente en el perioperatorio, así como el paciente, estén al tanto de las posibles complicaciones

\section{Los autores declaran no tener conflicto de interés.}




\section{REFERENCIAS}

1. Guilmette J, Sadow PM. Parathyroid Pathology. Surg Pathol Clin. 2019; 12 (4): 1007-1019. https://doi.org/10.1016/j.path.2019.08.006

2. Reid LM, Kamani D, Randoplh GW. Management of Parathyroid Disorders. Cummings Otolaryngology Head and Neck Surgery. 7ma edición. Filadelfia, Pensilvania: Elsevier: 2020. 1899-1916.

3. Demir AD. A review of parathyroid mass and patients with nonspecific complaints. J Int Med $\begin{array}{llll}\text { Res. } 2020 ; & 0 & (0): & 1-6 .\end{array}$ https://doi.org/10.1177/0300060519827169

4. Jason DS, Balentine CJ. Intraoperative Decision Making in Parathyroid Surgery. Surg Clin North Am. 2019; 99 (4): 681-691. https://doi.org/10.1016/j.suc.2019.04.008

5. Baj J, Sitarz R, Łokaj M, Forma A, Czeczelewski M, Maani A, et al. Preoperative and Intraoperative Methods of Parathyroid Gland Localization and the Diagnosis of Parathyroid Adenomas. Molecules [Internet]. MDPI AG; 2020 Apr 9;25(7):1724. Available from: http://dx.doi.org/10.3390/molecules25071724

6. Goltzman D. Phisiology of Parathyroid Hormon. Endocrinol Metab Clin North Am. 2018; 47 (4): 743-758. https://doi.org/10.1016/i.ecl.2018.07.003

7. Bringhurst FR. Demay MB, Kronenberg HM. Hormones and Disorders of Mineral Metabolism. Williams Textbook of Endocrinology. 14va edición. Filadelfia, Pensilvania: Elsevier; 2020. 1196-1255.

8. Rao SD. Epidemiology of Parathyroid disorders. Best Pract Clin Endocrinol Metab. 2018; 32 (6): 773-780. https://doi.org/10.1016/j.beem.2018.12.003

9. Kuzminski S, Sosa JA, Hoang JK. Update in Parathyroid Imaging. Magn Reson Imaging Clin N Am. $2018 ; \quad 26$ (1): 151-166. https://doi.org/10.1016/j.mric.2017.08.009
10. Shoback DM, Schafer AL, Bikle DD. Metabolic Bone Disease. Greenspan's Basic and Clinical Endocrinology. 10ma edición. Nueva York, Estados Unidos: McGraw- Hill: 2017. 239-297.

11. Sethi N, England RJ. Parathyroid surgery:from inception to the modern day. $\mathrm{Br} \mathrm{J}$ Hosp Med $\begin{array}{llll}\text { (Lond). } \quad 2017 ; & 78 & \text { (6): } 333-337 .\end{array}$ https://doi.org/10.12968/hmed.2017.78.6.333

12. Walker MD, Silverberg SJ. Primary hyperparathyroidism. Nat Rev Endocrinol. 2018; $14 \quad$ (2): 115-125. https://doi.org/10.1038/nrendo.2017.104

13. Insogna KL. Primary hyperparathyroidism. N Eng J Med. 2018; 379 (11): 1050-1059. https://doi.org/10.1056/NEJMcp1714213

14. Fuleihan GE, Arnold A. Pathogenesis and etiology of primary hyperparathyroidism. 2020. Uptodate. Disponible en: https://www-uptodatecom.binasss.idm.oclc.org/contents/pathogenesisand-etiology-of-primaryhyperparathyroidism?search=pathogenesis $\% 20$ a nd\%20etiology\%20of\%20primary\%20hyperparat hyroidism\&source $=$ search result\&selectedTitle $=1$ 120\&usage type=default\&display rank $=1$

15. Zhu CY, Sturgeon C, Yeh MW. Diagnosis and Management of Primary Hyperparathyroidism. JAMA. $2020 ; \quad 323$ (12): 1186-1187. https://doi.org/10.1001/jama.2020.0538

16. Mc Henry CR, Shi HH. Can parathyroid hyperplasia be predicted preoperatively?. Am J $\begin{array}{llll}\text { Surg. } \quad 2018 ; & 215 & \text { (3): } 389-392 .\end{array}$ https://doi.org/10.1016/.amjsurg.2017.10.051

17. Goswamy J, Lei M, Simo R. Parathyroid carcinoma. Curr Opin Otolaryngol Head Neck

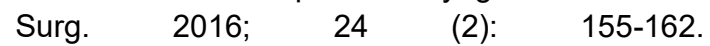
https://doi.org/10.1097/MO0.000000000000023

18. Cetani F, Pardi E, Marcocci C. Parathyroid Carcinoma. From Hom Res. 2019; 51: 63-76. https://doi.org/10.1159/000491039 